2 DR ADA S CHEUNG (Orcid ID : 0000-0001-5257-5525)

3 PROFESSOR BU BENG YEAP (Orcid ID : 0000-0002-7612-5892)

4 DR MATHIS GROSSMANN (Orcid ID : 0000-0001-8261-3457)

Article type $\quad$ : 3 Original Article - Australia, Japan, SE Asia

\title{
Effects of androgen deprivation therapy on telomere length.
}

Ada S. Cheung1,2, Bu B. Yeap ${ }^{3,4}$, Rudolf Hoermann"1, Jennie Hui ${ }^{5}$, John P. Beilby ${ }^{5}$, Mathis

${ }^{1}$ Department of Medicine, The University of Melbourne, Melbourne, Victoria,

Australia. 2Department of Endocrinology, Austin Health, Melbourne, Victoria,

Australia. ${ }^{3}$ School of Medicine and Pharmacology, University of Western Australia,

Perth, Western Australia, Australia. ${ }^{4}$ Department of Endocrinology and Diabetes, Fiona

Stanley Hospital, Perth, Western Australia, Australia. ${ }^{5}$ PathWest Laboratory Medicine,

Sir Charles Gairdner Hospital, Perth, Western Australia.

Short title: androgen deprivation and telomeres

Correspondence: Dr. Mathis Grossmann, Austin Health, Studley Road, Heidelberg,

23 Victoria, 3084, Australia. Tel +613 9496 5489; Fax +613 9496 2980;

24 Email: mathisg@unimelb.edu.au

Keywords (MeSH): Androgens, telomeres, testosterone, oestradiol

27

Word Count: Abstract 186; Manuscript text 1,997; Tables: 2; Figures 2.

Disclosure summaries: The authors have nothing to disclose.

This is the author manuscript accepted for publication and has undergone full peer review but has not been through the copyediting, typesetting, pagination and proofreading process, which may lead to differences between this version and the Version of Record. Please cite this article as doi: $10.1111 /$ cen.13382

This article is protected by copyright. All rights reserved 
Abstract

33 Objective: Recent evidence suggests that androgens either directly or via aromatisation

34 to oestradiol may regulate telomere length, hence providing a mechanism whereby

35 reproductive steroids are linked to biological aging in men. Using men with prostate cancer initiating androgen deprivation therapy (ADT), we tested the hypothesis that severe sex steroid deprivation would accelerate telomere shortening.

Design: We conducted a secondary analysis of a 2-year prospective controlled study among 65 men with non-metastatic prostate cancer newly commencing adjuvant ADT $(n=40)$ and age- and radiotherapy-matched prostate cancer controls $(n=25)$.

Methods: We measured leukocyte telomere length (LTL) expressed as telomeric/single copy control gene (T/S) ratio at baseline, 6, 12 and 24 months. Generalised linear models determined the mean adjusted difference (MAD) [95\% confidence interval] between groups during follow-up.

Results: Compared to controls over 24 months, men receiving ADT had no change in LTL, MAD for T/S ratio (0.105 [-0.004; 0.213], p=0.235).

Conclusions: Using men with prostate cancer receiving ADT as a model we found no evidence that prolonged and profound sex steroid deprivation is associated with accelerated telomerase shortening. Larger studies will be required to confirm, or refute these findings.

\section{Introduction}

58 Telomeres are specialised hexanucleotide repeats complexed to proteins. They are

59 located at ends of linear chromosomes and protect their structural integrity ${ }^{1}$. Telomere 60 length reduces with increasing chronological age and with accumulation of age-related comorbidities. Given that telomere shortening contributes to cellular senescence, telomere length represents a cellular marker of biological aging ${ }^{1}$. Recent preclinical,

63 observational and interventional studies suggest that androgens play a role in 
64 preserving telomere length $2-5$. However, whether this is an androgen receptor mediated effect, or whether androgens act indirectly, via aromatisation to oestradiol 6,7, 66 is unclear.

67

Because prostate cancer is an androgen responsive malignancy, androgen deprivation therapy (ADT, defined here as medical castration using gonadotropin-releasing hormone (GnRH) analogs) is an effective treatment. ADT reduces serum testosterone, but also oestradiol levels to castrate range. ADT is often prescribed for extended times (e.g. 3 years for high-risk prostate cancer with curative intent). Therefore, these patients offer a unique model of profound and global sex steroid deficiency over an extended period ${ }^{8}$ : ADT represents the only situation where an ethical requirement for $\mathrm{T}$ replacement is absent for prolonged periods. Given that ADT reduces both circulating testosterone and estradiol to near castrate levels, effects on telomere length -if testosterone indeed plays a role- should be evident irrespective of whether this occurs as a direct effect or via its aromatization to estradiol. Moreover, ADT also accelerates certain features associated with biological aging, such as loss of bone ${ }^{9}$ and muscle mass ${ }^{10}$, and increased insulin resistance ${ }^{11}$. We therefore conducted a secondary analysis of a 2-year, prospective case-control study in men with prostate cancer initiating ADT adjuvant to radiotherapy and age- and radiotherapy-matched prostate cancer controls to test the hypothesis that sex steroid deprivation accelerates telomere shortening.

\section{Subjects and Methods}

We conducted a prospective 24-month case-control study at a tertiary referral hospital (Austin Health, Melbourne, Australia). The study was approved by the Human Research Ethics Committee, Austin Health. All participants provided written informed consent. This is a secondary analysis assessing the effects of ADT on telomere length. The effects of ADT on muscle function, the primary outcome of the study are reported elsewhere 12 .

93 Participants were recruited from prostate cancer outpatient clinics. Inclusion criteria included age 55-85 years, localised non-metastatic prostate cancer (Stage T1-3, Nx, M0), and an Eastern Co-operative Oncology Group performance status of 0 (fully active and unrestricted in physical activity). Exclusion criteria included that any illnesses or other 
factors predisposing them to androgen deficiency, previous ADT, or significant medical comorbidities including active renal, liver, cardiac, respiratory or joint or neuromuscular disease. Cases were newly commencing long-term ADT with different brands of GnRH agonists, including triptorelin, goserelin, eligard and lucrin, at the discretion of the treating physician. To assess the specific effects of ADT, cases and controls were matched for age, body mass index, medical co-morbidities, radiotherapy treatment and baseline testosterone level ${ }^{12}$.

104

Blood was drawn in the morning and in the fasted state at 0, 6, 12 and 24 months. Serum total testosterone was measured with a electrochemiluminescence immunoassay using Cobas C8000, Roche Diagnostics (minimum detection $0.4 \mathrm{nmol} / \mathrm{l}$, inter-assay variation 5.0-6.9\%), as described 12. Oestradiol was measured by electrochemiluminescence immunoassay using the same system (minimum detection $19.0 \mathrm{pmol} / \mathrm{l}$, inter-assay variation $1.9-3.5 \%)$.

Leukocyte telomere length (LTL) was measured from leukocyte DNA samples by a 113 multiplex quantitative PCR method as described 5. We optimized a PCR-based 114 methodology for accurate measurement of LTL using the protocol described by 115 Cawthon 13 which we have further developed 5. Briefly, telomere lengths of leukocyte 116 DNA samples were measured by a multiplex quantitative PCR method. Each sample was 117 amplified for telomeric DNA and for beta globin, a single-copy control gene, which was 118 used as an internal control to normalize the starting amount of DNA. The K562 cell line 119 was used as a standard ${ }^{14}$. A four-point standard curve derived from the K562 cell line 120 was included in each run to assess and compensate for interplate variations in PCR 121 efficiency. The mean PCR efficiencies for telomeric DNA and beta globin are 94.5\% and 122 92.4\% respectively. Two quality-control samples were also included in each run to 123 assess interplate and intraplate variability of threshold cycle (Ct) values. Furthermore, $1245 \%$ of the test samples were repeated on a different run to account for interplate 125 variability. Periodic reproducibility experiments were performed to confirm adequate 126 normalization. All samples, standards, and controls were run in triplicate and the 127 median value used for analyses. A standard curve derived from K562 cell line was used 128 to transform the cycle threshold into nanograms of DNA. The amount of telomeric DNA 129 (T) was divided by the amount of single-copy control gene DNA (S), producing a relative 
measurement of the telomere length (T/S ratio), representing the normalised quantity

131 of telomeric DNA. The coefficient of variation for the quantitative PCR across batches

132 was $<10 \%$.

133 Statistical analysis

134 Data were not normally distributed and are presented as median and interquartile 135 range (IQR). Comparisons of baseline characteristics were made using Wilcoxon rank 136 sum test for continuous variables or chi square test for frequencies. Two sided p values $137<0.05$ were considered significant. Repeated measurements were compared between 138 groups using a linear mixed model ${ }^{15}$. The effect of interest was the interaction of time 139 points and group, incorporating baseline values as a fixed covariate and repeated 140 measure by subject as random effect. The model is also robust against regression to the 141 mean. As a quantitative measure, mean adjusted difference (MAD) plus 95\% CI between 142 the groups from baseline to 24 months is provided. Statistical analyses were performed 143 using R statistical package (version 3.3.2 for Mac) ${ }^{16}$.

\section{Results}

146 Baseline characteristics of the study subjects

147 Study participants were matched for age, body mass index, medical co-morbidities, 148 radiotherapy and baseline testosterone level (Table 1). Given ADT is added to 149 radiotherapy as treatment for high-risk disease, whereas radiotherapy alone is 150 indicated for intermediate risk disease, baseline Gleason scores and PSA levels were 151 higher in cases compared to controls. At baseline, all men were clinically eugonadal and 152 had age-appropriate normal testosterone levels. There was no difference in baseline 153 LTL, measured by the T/S ratio between cases and controls (Table 1).

155 While serum total testosterone and oestradiol levels remained stable in controls, both 156 were decreased to castrate levels in men receiving ADT over the course of the study 157 (Figure 1). Only men recruited relatively early in the study had assessments at 24 158 months, because men recruited al later time points were not followed beyond 12 159 months due to funding limitations.

160 
162 There was no difference in the LTL, measured by the T/S ratio and presented as mean 163 adjusted differences at each time point of follow-up (6, 12 and 24 months) between 164 men receiving ADT and the age- and radiotherapy-matched prostate cancer, overall $165 \mathrm{p}=0.235$ (Table 2, Figure 2).

\section{Discussion}

168 In this controlled prospective study, using men with prostate cancer receiving ADT as a 169 model, we found no evidence that severe sex steroid deprivation over 2 years is 170 associated with shorter telomere length.

172 Our analysis was prompted by recent evidence that sex steroids may modulate telomere 173 length, a marker of cellular senescence, hence providing a potential mechanisms linking 174 decreasing levels of circulating sex steroids to biological aging in men. In a recent cross175 sectional study of community dwelling men, we reported that circulating levels of the 176 testosterone metabolites dihydrotestosterone (DHT) and oestradiol, but not 177 testosterone itself, correlate with LTL independently of age ${ }^{5}$. In experimental studies 178 using a variety of cultured cells, testosterone ${ }^{3}$, synthetic androgens ${ }^{2}$ and oestradiol 2, 6, 7 179 have been reported to up-regulate telomerase activity, the enzyme that counters 180 telomere shortening. This occurs by sex steroids increasing the expression of TERT, the gene expressing the catalytic subunit of telomerase $2,6,7$. This effect may involve the activation of an estrogen-responsive element in the reporter region of the telomerase gene ${ }^{2,6}$. Therefore, based on these preclinical studies, it has been assumed that the aromatisation of androgens to oestradiol is important for telomerase up-regulation. This is consistent with our observational Mendelian randomisation study reporting an association between aromatase gene polymorphisms reducing serum oestradiol and shorter LTL in community dwelling men ${ }^{5}$. However, in a recent phase 1-2 prospective study, danazol treatment, a non-aromatisable androgen that cannot be converted to oestradiol 17, has been reported to prevent telomerase shortening in patients with genetic defects in telomere maintenance and repair ${ }^{4}$. This suggests that aromatization to an estrogen capable of activating an estrogen receptor does not appear to be necessary for the effect of danazol on telomere length ${ }^{18}$. Given that either androgens, 193 oestrogens or both may be involved in the regulation of telomere length, we studied 194 men receiving ADT, as ADT reduces both testosterone and its metabolites DHT and 
195 oestradiol to castrate levels. Moreover, ADT promotes the development of 196 sarcopaenia ${ }^{10}$, of structural bone decay 9 and of insulin resistance ${ }^{11}$, all features 197 associated with biological aging in men. Given the negative findings of our study, further 198 work is required to determine whether telomere shortening plays a role, if any, in 199 promoting ADT-associated adverse effects resembling that of accelerated aging.

201 To date, clinical studies assessing the effects of sex steroids in telomere length have 202 been limited to cross-sectional studies among community dwelling men ${ }^{5,19}$. This study, 203 to our knowledge, constitutes the first longitudinal controlled study examining the 204 effects of changes in circulating sex steroids on telomere length. Only ADT-naïve men 205 without clinical androgen deficiency who had, prior to commencing ADT, normal 206 circulating testosterone levels were recruited. The focus on men receiving ADT ensured 207 that both testosterone and oestradiol were reduced to castrate levels. In addition, the 208 inclusion of an age- and radiotherapy and comorbidity- matched control group 209 facilitated the delineation of specific effects of ADT. In addition, men were relatively 210 healthy, living in the community without functional impairment receiving adjuvant ADT 211 with curative intent, and all participants maintained undetectable prostate specific 212 antigen levels throughout the study ${ }^{12}$.

214 The main limitation of the study is its relatively small sample size, especially with 215 regards to the 2 year time point, and hence this study lacks the power to detect modest 216 effects on telomere length. Indeed, this is a secondary analysis of a study powered to 217 detect the effect of ADT on biomechanical leg muscle function ${ }^{12}$. In addition, 2 years is a 218 relatively short time frame, and a longer duration may well be necessary to detect a 219 significant effect of sex steroid deprivation. However, the severe reduction of sex 220 steroid levels following ADT is much more rapid and profound in magnitude than the 221 age-related decline in testosterone levels among community dwelling men not receiving 222 ADT 20. The study was not powered to assess whether changes in telomere length 223 during ADT are associated with accelerated clinical features of aging observed during 224 ADT, such as loss of muscle mass and function 10,12 , loss of bone mass ${ }^{9}$ or metabolically 225 unfavourable changes in body composition ${ }^{11,21}$. 
227 While we did not use Southern-blot based quantification of telomere length, the qPCR228 based methodology used here has been reported to be strongly correlated to Southern229 blot methodology with reported correlation coefficients of ranging from $r=0.84$ to $230>0.9$ 13,22. To minimize measurement error of LTL over time, care was taken to ensure 231 that all samples were extracted and processed by the same technician using the same 232 protocol in one laboratory.

233 Exposure to significant ionizing radiation has been reported to be inversely correlated 234 with LTL 23. In the absence of a control group not receiving radiation, we cannot 235 discount possible effects of pelvic irradiation on telomere length. However, given that 236 our objective was to assess the effects of sex steroid deprivation on telomere length, inclusion of a radiotherapy-matched control group should have eliminated possible confounding effects of radiation treatment.

In conclusion, in this 2-year prospective case controlled study we found no evidence that severe sex steroid deprivation accelerates telomerase shortening in men who have been diagnosed with prostate cancer. However larger, longer term studies are required to confirm, or to refute these findings.
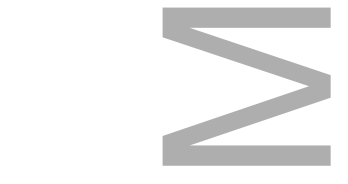

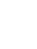

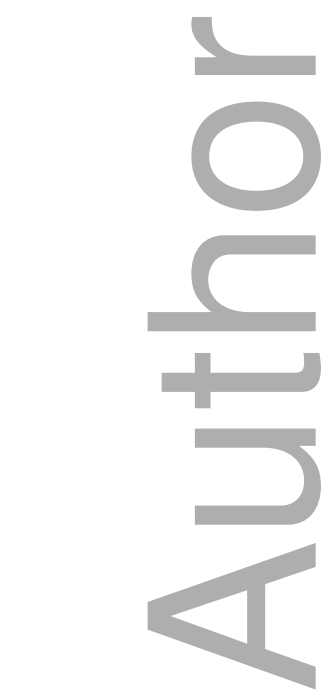

\section{Declaration of interest:}


259 There was no conflict of interest that could be perceived as prejudicing the impartiality

260 of the research reported.

261

262 Acknowledgement:

263 We would like to thank Gillian M. Arscott, PathWest Laboratory Medicine, Sir Charles

264 Gairdner Hospital, Perth, Western Australia, for assistance with conducting the

265 leukocyte telomere length measurements.

266

267 Funding:

268 The work was supported by the National Health and Medical Research Council of 269 Australia (grant numbers \#1006407, \#1017233 and \#1024139).

270

271 Author Contribution statement:

272 A.S. researched data and wrote the manuscript, R.H. researched data, contributed to the

273 discussion and reviewed/edited the manuscript. B.B.Y. researched data and reviewed/

274 edited the manuscript. J.H. researched data and reviewed/ edited the manuscript J.P.B.

275 researched data and reviewed/ edited the manuscript. M.G. designed the study,

276 researched data and wrote the manuscript. A.S. and M.G. are the guarantors for the

277 study.

278

279

280

281

282

283 Figure Legends

284 Figure 1. Circulating total testosterone and oestradiol levels in men receiving ADT and

285 matched controls

286 Shown are serum total testosterone (Figure 1a) and oestradiol levels (Figure 1b)

287 (adjusted mean, 95\% CI) in men with prostate cancer receiving ADT (continuous line)

288 and aged and radiotherapy matched prostate cancer controls (dashed line).

289 In cases were sex steroids levels were undetectable, levels were set at the lower limit of 290 detection, $0.4 \mathrm{nmol} / \mathrm{l}$ for total testosterone, and $19 \mathrm{pmol} / \mathrm{l}$ for oestradiol respectively. $\mathrm{P}$

291 values refer to the overall significance of the change between groups during follow-up. 
293 Figure 2. Leukocyte telomere length in men receiving ADT and matched controls

294 Shown are leukocyte telomere lengths, expressed as T/S ratio (adjusted mean, 95\% CI)

295 in men with prostate cancer receiving ADT (continuous line) and aged and radiotherapy

296 matched prostate cancer controls (dashed line). The $\mathrm{P}$ value refers to the overall 297 significance of the change between groups during follow-up.

\section{References}

2991 Blackburn, E.H., Greider, C.W. \& Szostak, J.W. (2006) Telomeres and telomerase: 300 the path from maize, Tetrahymena and yeast to human cancer and aging. Nat Med 12, $301 \quad 1133-1138$.

3022 Calado, R.T., Yewdell, W.T., Wilkerson, K.L., Regal, J.A., Kajigaya, S., Stratakis, C.A. 303 \& Young, N.S. (2009) Sex hormones, acting on the TERT gene, increase telomerase 304 activity in human primary hematopoietic cells. Blood 114, 2236-2243.

3053 Nourbakhsh, M., Golestani, A., Zahrai, M., Modarressi, M.H., Malekpour, Z. \& 306 Karami-Tehrani, F. (2010) Androgens stimulate telomerase expression, activity and 307 phosphorylation in ovarian adenocarcinoma cells. Mol Cell Endocrinol 330, 10-16.

3084 Townsley, D.M., Dumitriu, B., Liu, D., Biancotto, A., Weinstein, B., Chen, C., Hardy, 309 N., Mihalek, A.D., Lingala, S., Kim, Y.J., Yao, J., Jones, E., Gochuico, B.R., Heller, T., Wu, C.O., 310 Calado, R.T., Scheinberg, P. \& Young, N.S. (2016) Danazol Treatment for Telomere 311 Diseases. NEnglJ Med 374, 1922-1931.

3125 Yeap, B.B., Knuiman, M.W., Divitini, M.L., Hui, J., Arscott, G.M., Handelsman, D.J., 313 McLennan, S.V., Twigg, S.M., McQuillan, B., Hung, J. \& Beilby, J.P. (2016) Epidemiological 314 and Mendelian Randomization Studies of Dihydrotestosterone and Estradiol and 315 Leukocyte Telomere Length in Men. J Clin Endocrinol Metab 101, 1299-1306.

3166 Kyo, S., Takakura, M., Kanaya, T., Zhuo, W., Fujimoto, K., Nishio, Y., Orimo, A. \& 317 Inoue, M. (1999) Estrogen activates telomerase. Cancer Res 59, 5917-5921.

3187 Nanni, S., Narducci, M., Della Pietra, L., Moretti, F., Grasselli, A., De Carli, P., Sacchi, 319 A., Pontecorvi, A. \& Farsetti, A. (2002) Signaling through estrogen receptors modulates 320 telomerase activity in human prostate cancer. J Clin Invest 110, 219-227.

3218 Grossmann, M., Cheung, A.S. \& Zajac, J.D. (2013) Androgens and prostate cancer; 322 pathogenesis and deprivation therapy. Best Pract Res Clin Endocrinol Metab 27, 603323616. 
3249 Hamilton, E.J., Ghasem-Zadeh, A., Gianatti, E., Lim-Joon, D., Bolton, D., Zebaze, R., 325 Seeman, E., Zajac, J.D. \& Grossmann, M. (2010) Structural decay of bone 326 microarchitecture in men with prostate cancer treated with androgen deprivation 327 therapy. JClin Endocrinol Metab 95, E456-463.

32810 Smith, M.R., Saad, F., Egerdie, B., Sieber, P.R., Tammela, T.L., Ke, C., Leder, B.Z. \& 329 Goessl, C. (2012) Sarcopenia during androgen-deprivation therapy for prostate cancer. J 330 Clin Oncol30, 3271-3276.

33111 Basaria, S., Muller, D.C., Carducci, M.A., Egan, J. \& Dobs, A.S. (2006) 332 Hyperglycemia and insulin resistance in men with prostate carcinoma who receive 333 androgen-deprivation therapy. Cancer 106, 581-588.

33412 Cheung, A.S., Gray, H., Schache, A.G., Hoermann, R., Lim Joon, D., Zajac, J.D., Pandy, 335 M.G. \& Grossmann, M. (2017) Androgen deprivation causes selective deficits in the 336 biomechanical leg muscle function of men during walking: a prospective case-control 337 study. J Cachexia Sarcopenia Muscle 8, 102-112.

33813 Cawthon, R.M. (2009) Telomere length measurement by a novel monochrome 339 multiplex quantitative PCR method. Nucleic Acids Res 37, e21.

34014 Codd, V., Mangino, M., van der Harst, P., Braund, P.S., Kaiser, M., Beveridge, A.J., 341 Rafelt, S., Moore, J., Nelson, C., Soranzo, N., Zhai, G., Valdes, A.M., Blackburn, H., Mateo 342 Leach, I., de Boer, R.A., Kimura, M., Aviv, A., Wellcome Trust Case Control, C., Goodall, 343 A.H., Ouwehand, W., van Veldhuisen, D.J., van Gilst, W.H., Navis, G., Burton, P.R., Tobin, 344 M.D., Hall, A.S., Thompson, J.R., Spector, T. \& Samani, N.J. (2010) Common variants near 345 TERC are associated with mean telomere length. Nat Genet 42, 197-199.

34615 Bates, D., Maechler, M., Bolker, B. \& Walker, S. (2015) Fitting Linear Mixed347 Effects Models Using lme4. Journal of Statistical Software 67, 1-48.

34816 R Core Team (2016) R: A language and environment for statistical computing. R 349 Foundation for Statistical Computing, Vienna, Austria.

35017 de Oca Porto, R.M., Fernandez, A.R., Brito, D.M., Vidal, T.C. \& Diaz, A.L. (2006) Gas 351 chromatography/mass spectrometry characterization of urinary metabolites of danazol 352 after oral administration in human. J Chromatogr B Analyt Technol Biomed Life Sci 830, 353 178-183.

35418 Grossmann, M. (2016) Danazol Treatment for Telomere Diseases. $N$ Engl J Med $355375,1095$. 
35619 Bekaert, S., Van Pottelbergh, I., De Meyer, T., Zmierczak, H., Kaufman, J.M., Van 357 Oostveldt, P. \& Goemaere, S. (2005) Telomere length versus hormonal and bone mineral 358 status in healthy elderly men. Mech Ageing Dev126, 1115-1122.

35920 Harman, S.M., Metter, E.J., Tobin, J.D., Pearson, J. \& Blackman, M.R. (2001) 360 Longitudinal effects of aging on serum total and free testosterone levels in healthy men. 361 Baltimore Longitudinal Study of Aging. J Clin Endocrinol Metab 86, 724-731.

36221 Cheung, A.S., Hoermann, R., Dupuis, P., Joon, D.L., Zajac, J.D. \& Grossmann, M. 363 (2016) Relationships between insulin resistance and frailty with body composition and 364 testosterone in men undergoing androgen deprivation therapy for prostate cancer. EurJ 365 Endocrinol175,229-237.

36622 Aviv, A., Hunt S.C., Lin J., Cao X., Kimura M., Blackburn E. (2011) Impartial 367 comparative analysis of measurement of leukocyte telomere length/DNA content by 368 Southern blots and qPCR. Nucleic Acids Res 39, e134.

36923 Lustig A., Shterev I., Geyer S., Shi A., Hu Y., Morishita Y., Nagamura H., Sasaki K., 370 Maki M., Hayashi I., Furukawa K., Yoshida K., Kajimura J., Kyoizumi S., Kusunoki Y., 371 Ohishi W., Nakachi K., Weng N.P., Hayashi T. (2016) Long term effects of radiation 372 exposure on telomere lengths of leukocytes and its associated biomarkers among 373 atomic-bomb survivors. Oncotarget 7, 38988-38998.

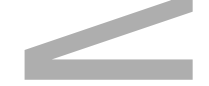


Table 1. Baseline characteristics

\begin{tabular}{|l|c|c|c|}
\hline Baseline Characteristic & ADT group N=40 & Control group N=25 & p value \\
\hline Age & $67.0[61.3 ; 73.3]$ & $70.6[65 ; 73.3]$ & 0.21 \\
\hline Prostate Cancer Gleason Score & $9[7 ; 9]$ & $7[7 ; 7]$ & $<0.01$ \\
\hline Concurrent radiotherapy treatment & $91.2 \%$ & $88.9 \%$ & 0.74 \\
\hline Previous prostatectomy & $54.3 \%$ & $77.8 \%$ & 0.06 \\
\hline Total testosterone (nmol/L) & $13.2[9.2 ; 18.5]$ & $15.5[11.1 ; 17.0]$ & 0.55 \\
\hline PSA (ug/L) & $2.0[0.16 ; 12.8]$ & $0.07[0.03 ; 0.35]$ & $<0.01$ \\
\hline Charlson co-morbidity index & $2[2 ; 3]$ & $3[2 ; 3]$ & 0.17 \\
\hline Medical comorbidities & & & \\
\hline Ischaemic heart disease & $26.5 \%$ & $14.8 \%$ & 0.30 \\
\hline Diabetes mellitus & $17.6 \%$ & $18.5 \%$ & 0.89 \\
\hline Liver disease & $0 \%$ & $0 \%$ & $>0.99$ \\
\hline Chronic kidney disease & $0 \%$ & $0 \%$ & $>0.99$ \\
\hline Hypertension & $55.9 \%$ & $59.3 \%$ & 0.70 \\
\hline LTL, T/S ratio & $1.01[0.89 ; 1.13]$ & $0.94[0.86 ; 1.04]$ & 0.106 \\
\hline
\end{tabular}

399 PSA = prostate specific antigen; $\mathrm{LTL}=$ leukocyte telomere length; $\mathrm{T} / \mathrm{S}=$ telomeric DNA/ 400 single-copy control gene. Data presented are median [interquartile range] or 401 proportions (\%). Gleason score $<7=$ low-moderate risk, $7=$ intermediate risk, 8-10 = 402 high risk prostate cancer. 
Table 2. Leukocyte Telomere Length

\begin{tabular}{|l|l|l|l|l|l|l|}
\hline LTL, T/S ratio & ADT Group & $\mathbf{n}$ & Controls & $\mathbf{n}$ & $\begin{array}{l}\text { Mean adjusted } \\
\text { difference }[95 \% \mathrm{CI}]\end{array}$ & $\mathbf{p}$ value \\
\hline 0 months & $1.01[0.89 ; 1.13]$ & 40 & $0.94[0.86 ; 1.04]$ & 25 & & \\
\hline 6 months & $1.05[0.92 ; 1.19]$ & 35 & $0.95[0.90 ; 1.10]$ & 25 & $-0.006[-0.077 ; 0.069]$ & \\
\hline 12 months & $1.07[0.96 ; 1.22]$ & 28 & $1.03[0.94 ; 1.10]$ & 22 & $0.003[-0.072 ; 0.078]$ & \\
\hline 24 months & $1.06[0.99 ; 1.33]$ & 7 & $0.99[0.93 ; 1.11]$ & 12 & $0.105[-0.004 ; 0.213]$ & 0.235 \\
\hline
\end{tabular}

416

417

418 Medians [interquartile ranges] are presented. Mean adjusted difference refers to the

419 change over 12 months across groups (mixed model). $n$ denotes the number of study

420 subjects at each time point in each group. The $P$ value refers to the overall significance

421 of the change between groups during follow-up.

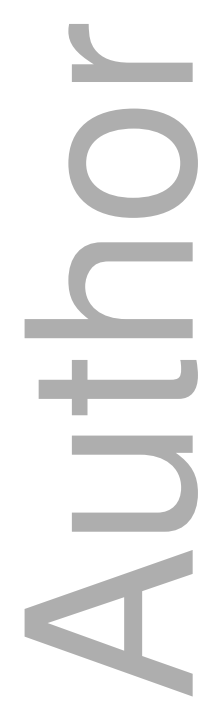




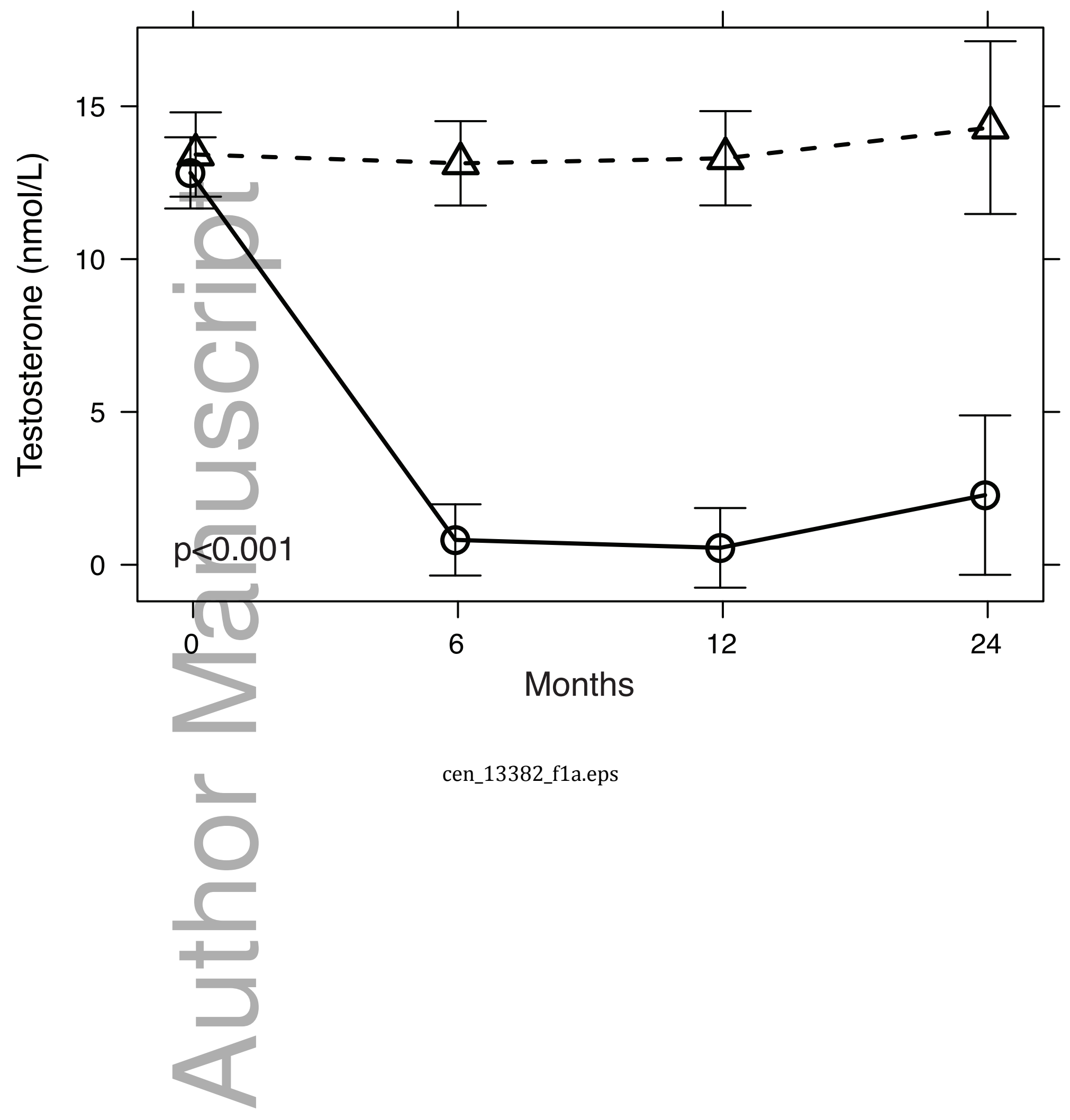

This article is protected by copyright. All rights reserved 


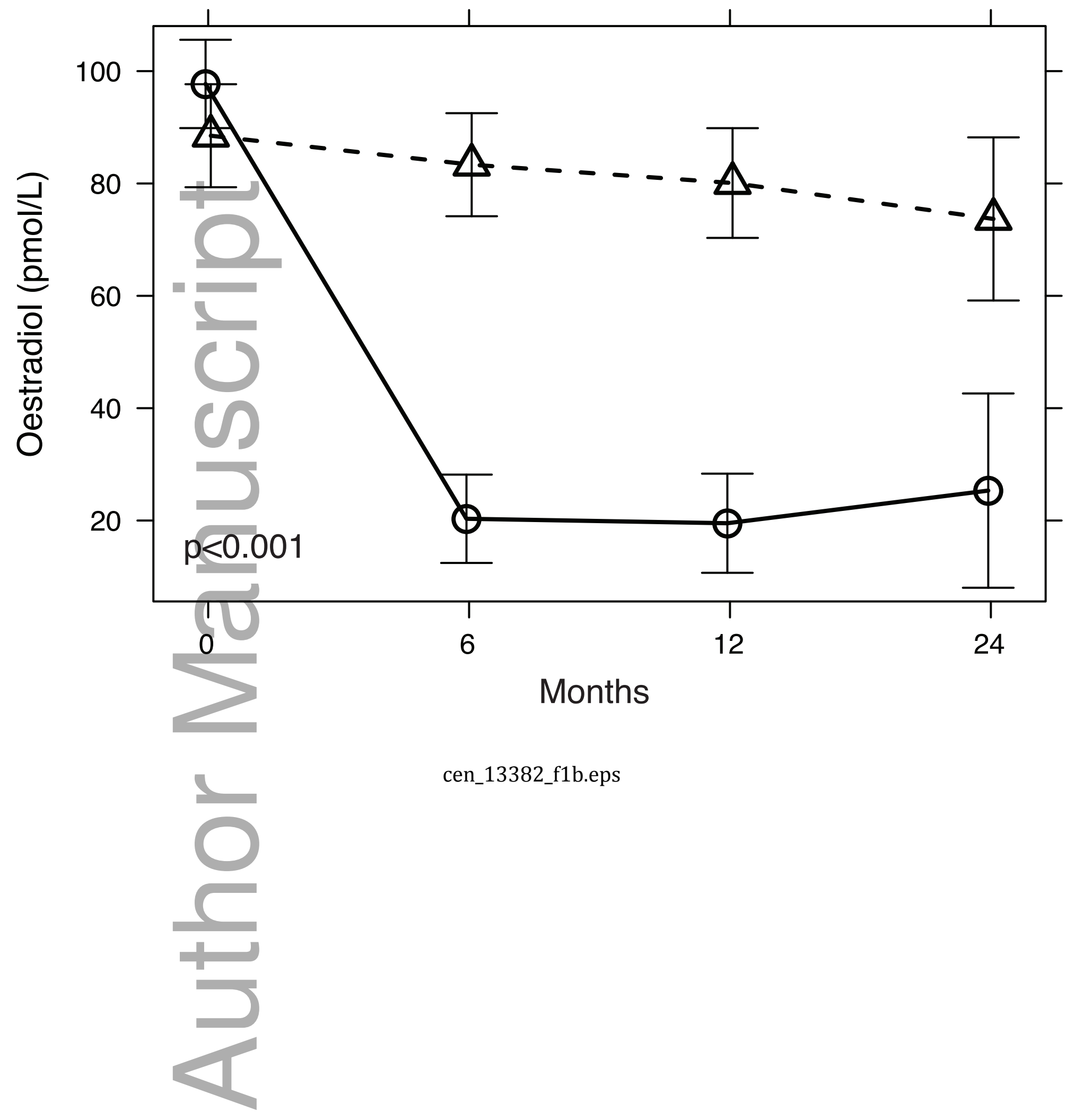

This article is protected by copyright. All rights reserved 


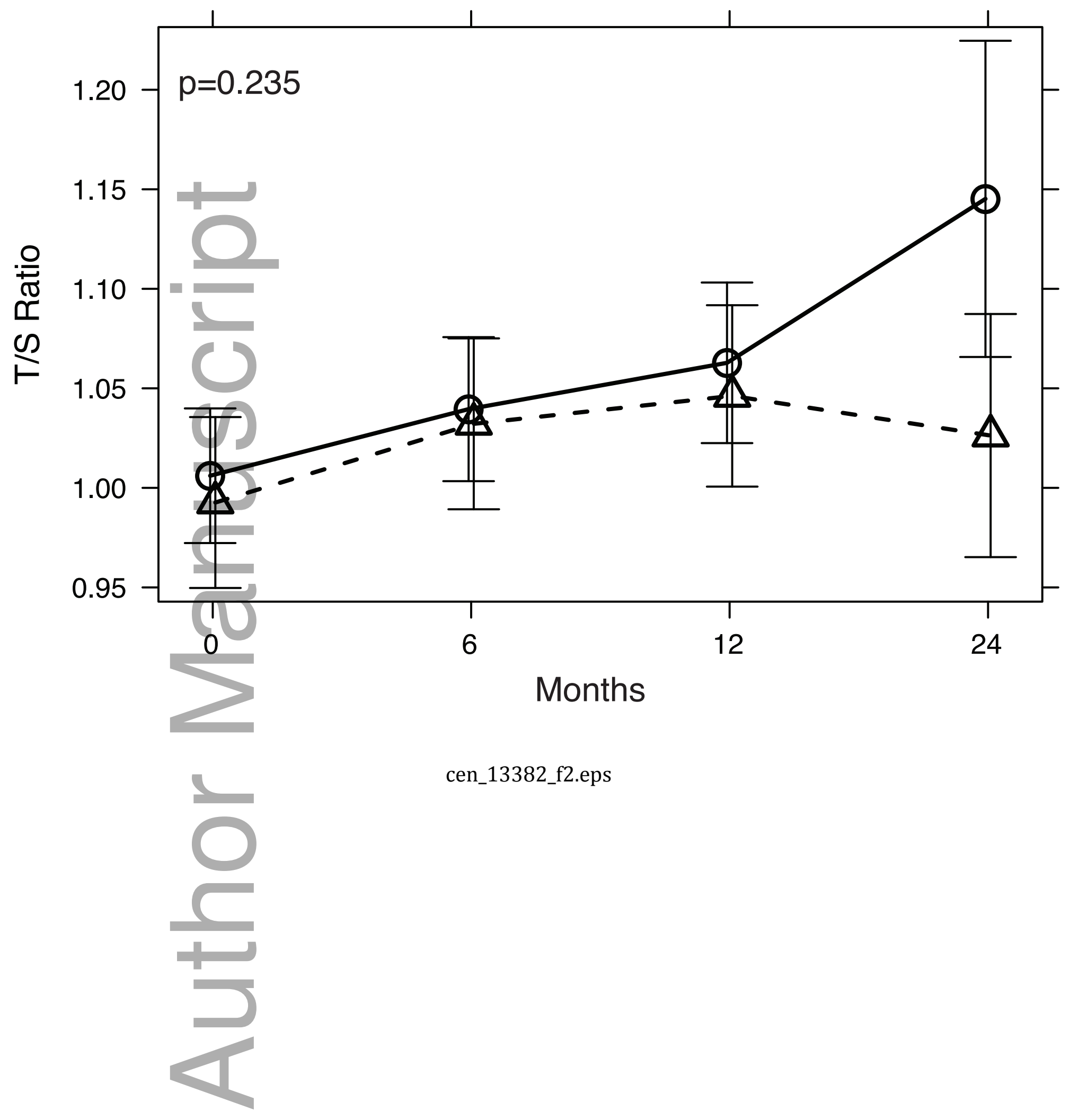

This article is protected by copyright. All rights reserved 


\section{University Library}

\section{- M M N E R VA A gateway to Melbourne's research publications}

Minerva Access is the Institutional Repository of The University of Melbourne

\section{Author/s:}

Cheung, AS;Yeap, BB;Hoermann, R;Hui, J;Beilby, JP;Grossmann, M

Title:

Effects of androgen deprivation therapy on telomere length

\section{Date:}

2017-10-01

Citation:

Cheung, A. S., Yeap, B. B., Hoermann, R., Hui, J., Beilby, J. P. \& Grossmann, M. (2017).

Effects of androgen deprivation therapy on telomere length. CLINICAL ENDOCRINOLOGY, 87 (4), pp.381-385. https://doi.org/10.1111/cen.13382.

Persistent Link:

http://hdl.handle.net/11343/293100 